\title{
STUDY ON OBSTETRICAL BRACHIAL PLEXUS PALSY IN RESPECT OF ELECTROPHYSIOLOGIC FEATURES AND FUNCTIONAL OUTCOME
}

\author{
V. Toopchizadeh ${ }^{1}$, M. Barzegar $^{2}$ \\ ${ }^{I}$ Physiacl Medicine and Rehabilitation Research Center, ${ }^{2}$ Pediatric Neurology, Tabriz University of Medical \\ Sciences, Tabriz, Iran
}

Background: An obstetric brachial plexus palsy (OBPP) is a traction lesion of the brachial plexus during delivery. Evaluating a patient with OBPP by electrodiagnostic methods is noninvasive, safe and accurate. This study aims at evaluating the OBPP in regard to electrophysiologic features and functional outcome.

Methods \& materials: In a cross-sectional analytic-descriptive setting, 42 child patients with OBPP were evaluated in Tabriz Children Hospital during a 13-month period. The patients' characteristics, as well as the electrophysiological findings were determined. Follow-up for at least until 2 year of age was reached in 28 $(66.7 \%)$ patients. The final outcome was determined in this group and the data were compared accordingly.

Results: Forty two patients, 25 females and 17 males were enrolled. Delivery was vaginal in $97.6 \%$ of cases, with assisted delivery only in 9.5\%. The most common fetal presentation was head (81\%). The Erb and Panplexus palsies were diagnosed in $71.4 \%$ and $28.6 \%$ of cases, respectively. Recovery was "good", "moderate" and "poor" in $53.6 \%, 35.7 \%$ and $10.7 \%$ of cases by the age of 2 years, respectively. On-admission electrodiagnostic study revealed a significantly higher rate of cases with decreased CMAP of ulnar and median nerves, decreased SNAP of median nerve and abnormal recruitments of thenar and extensor digitorum communis (EDC) muscles in patients with unacceptable recovery. The pan-plexus type of palsy was related with poor outcome.

Conclusion: According to our results, electrodiagnostic study can be helpful in perfecting outcome in patients with OBPP. Totally, over half of the patients had good recovery. 